\section{IN BRIEF}

\section{DIABETES}

\section{Reprogrammed $\alpha$-cells reverse diabetes}

Cellular reprogramming represents an attractive strategy to replenish the lost insulin-producing pancreatic $\beta$-cells in diabetes. Furuyama et al. now report that purified human islet $\alpha$ - and $\gamma$-cells transduced with PDX 1 and MAFA - transcription factors required for $\beta$-cell development, maturation and function - engage in insulin production in response to glucose. Transcriptomic and proteomic analysis of the modified $\alpha$-cells revealed expression signatures intermediate between $\alpha$ - and $\beta$-cells. Transplantation of reprogrammed human $\alpha$-cells into diabetic mice ameliorated clinical signs of diabetes.

ORIGINAL ARTICLE Furuyama, K. et al. Diabetes relief in mice by glucose-sensing insulin-secreting human $\alpha$-cells. Nature https://doi.org/10.1038/s41586-019-0942-8 (2019)

\section{$\Rightarrow$ NEURODEGENERATIVE DISEASE}

\section{Molecular chaperone treats encephalopathy}

Transmissible spongiform encephalopathies (TSEs) are fatal neurodegenerative disorders caused by infectious molecular variants of the host cell-encoded prion protein $(\operatorname{PrP})$, primarily the scrapie form, PrPSc. Yamaguchi et al. have designed a molecular chaperone (MC) that binds to and stabilizes normal PrP and eradicates PrPSc in infected cells, without the development of drug resistance. Weekly injection of prion-infected mice with $\mathrm{MC}$ prolonged survival. In bovine TSE-infected macaques, weekly intravenous MC treatment slowed disease onset and progression, and reduced neurological, physiological and behavioural abnormalities.

ORIGINAL ARTICLE Yamaguchi, K. et al. A designer molecular chaperone against transmissible spongiform encephalopathy slows disease progression in mice and macaques. Nat. Biomed. Eng. https://doi.org/10.1038/s41551-019-0349-8 (2019)

\section{CARDIOVASCULAR DISEASE}

\section{Sleep protects against atherosclerosis}

Lack of sleep increases the risk of developing a variety of diseases, but the underlying mechanisms remain unknown. Here, McAlpine et al. report that atherosclerosis-prone mice subjected to 16 weeks of sleep fragmentation (SF) display aggravated atherosclerosis, increased haematopoiesis and decreased hypothalamic production of the stimulatory neuropeptide hypocretin (Hcrt). In mice, Hcrt reduced haematopoiesis and suppressed atherosclerosis through the control of bone marrow colony-stimulating factor 1 (CSF1) production. Haematopoietic CSF1 deletion or Hcrt supplementation protected against SF-induced haematopoiesis and atherosclerosis in mouse models. ORIGINAL ARTICLE McAlpine, C. S. et al. Sleep modulates haematopoiesis and protects against atherosclerosis. Nature 566, 383-387 (2019)

\section{CANCER}

\section{Targeting polyamines in neuroblastoma}

Polyamines are critical for cell growth and survival and are commonly increased in MYCN-amplified cancers. However, clinical trials using single agents targeting the polyamine pathway have been largely disappointing. Gamble et al. show that MYCN directly modulates polyamine catabolism, synthesis and transport in neuroblastoma (NB). They identify the solute carrier family 3 member 2 as the key transporter involved in polyamine uptake in NB, which is regulated by MYCN. Inhibition of polyamine uptake with the small molecule AMXT-1501 in combination with difluoromethylornithine (an inhibitor of polyamine synthesis) effectively treated MYCN-amplified NB in transgenic and PDX mouse models. ORIGINAL ARTICLE Gamble, L. D. et al. Inhibition of polyamine synthesis and uptake reduces tumor progression and prolongs survival in mouse models of neuroblastoma. Sci. Transl Med. 11, eaau1099 (2019)

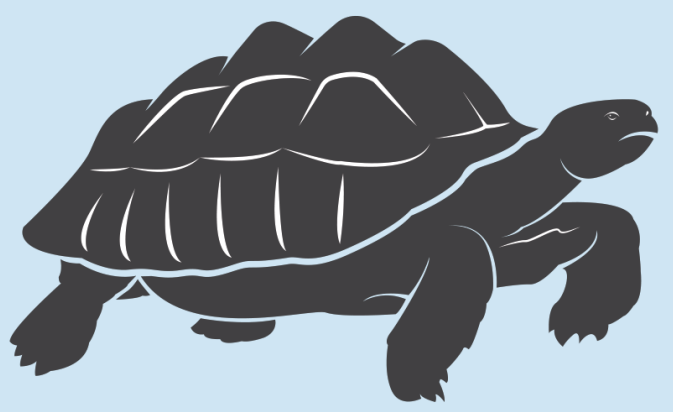

DRUG DELIVERY

\title{
Tortoise-inspired device for oral delivery of biologics
}

The delivery of biologics is largely limited to intravenous or subcutaneous injections. Oral delivery is more challenging to optimize but can improve patient compliance and therefore therapeutic efficacy. Langer, Traverso and colleagues have developed a new type of oral delivery system for biologics - the ingestible selforienting millimeter-scale applicator (SOMA) - and used it to deliver insulin through the stomach wall to achieve plasma levels similar to those from subcutaneous routes.

The SOMA design was inspired by the leopard tortoise shell, the shape and weight distribution of which helps tortoises right themselves if they flip over. A low centre of mass was achieved by using low-density, hollowed-out polycaprolactone for the upper curved portion of the SOMA and stainless steel on the bottom. Like the tortoise shell, the bottom of the SOMA is fairly flat and has distinct corners, which promotes reorienting when the device is off-centred.

Within this shell, the SOMA contains a $7 \mathrm{~mm}$ millipost consisting of a $1.7 \mathrm{~mm}$ tip $-0.5 \mathrm{mg}$ of insulin mixed with poly(ethylene)oxide under high pressure - and a shaft made of biodegradable polymers. The tip compression enabled insulin to be concentrated 100 times relative to aqueous solutions. Importantly, the insulin in the tip was stable at $40^{\circ} \mathrm{C}$ for 16 weeks.

The millipost needs to be deployed from the SOMA in the right location at the right time. To do this, the authors spring-loaded the millipost under the control of a hydration-dependent sucrose and isomalt actuator that also protects the payload from the gastric juices. Vents in the SOMA were made such that gastric juices would dissolve the barrier in the actuator and trigger millipost release.

Many orally delivered molecules are absorbed in the intestine, but the SOMA is designed to deliver its payload across the gastric wall, which has numerous advantages. First, the time to gastric emptying can be unpredictable, so the timing of delivery can be more tightly controlled with gastric delivery systems than with intestinal ones. Secondly, the gastric wall is much thicker $(4-6 \mathrm{~mm})$ than the intestinal wall $(0.1-0.2 \mathrm{~mm})$ and therefore provides a larger target for millipost insertion. The gastric wall also regenerates quickly and perforations are quickly sealed by mucous, so the gastric wall could be particularly safe for indications that require repeated administrations.

The SOMAs effectively delivered insulin and lowered blood glucose levels when administered to swine by endoscopy or when milliposts were surgically placed. The SOMA did not damage the stomach or cause any observable abnormalities. In principle, SOMAs containing milliposts with higher doses of insulin could be used for sustained delivery; such milliposts released their drug for 30 hours when implanted subcutaneously.

Although insulin is the most widely administered biologic, this delivery system could pave the way to oral delivery for numerous therapeutic agents including other proteins, antibodies or nucleic acids.

Megan Cully

ORIGINAL ARTICLE Abramson, A. et al. An ingestible self-orienting system for oral delivery of macromolecules. Science 363, 611-615 (2019) FURTHER READING Anselmo, A. C. et al. Non-invasive delivery strategies for biologics. Nat. Rev. Drug Discov. 18, 19-40 (2019) 